\title{
Systematic elucidation of the molecular mechanism of scutellarin against osteoarthritis based on network pharmacology
}

\section{Shijia Guo}

Shenyang Sport University

\section{Xinan Zhang}

Shenyang Sport University

mingli Sun ( $\square$ sun18940103126@163.com )

Shenyang Sport University https://orcid.org/0000-0001-5007-4241

\section{Research}

Keywords: Scutellarin, Osteoarthritis, Network pharmacology, Molecular docking, NFkB signaling pathway

Posted Date: July 21st, 2020

DOI: https://doi.org/10.21203/rs.3.rs-41443/v1

License: (c) (i) This work is licensed under a Creative Commons Attribution 4.0 International License.

Read Full License 


\section{Abstract \\ Background}

Scutellarin was reported to exerted inhibitive effects on osteoarthritis, However, the detailed mechanisms remain unclear. In this study, we investigated underlying multi-target mechanisms of scutellarin against osteoarthritis by using network pharmacology analysis and molecular docking.

\section{Results}

Scutellarin exerted inhibitive effects on osteoarthritis by regulating the function of several new signaling pathways, such as TNF signaling pathway, NOD-like receptor signaling pathway and HIF-1 signaling pathway. Molecular docking analysis showed there was better interaction between scutellarin and several NF-kB signaling proteins, including NFKBIA, RELA and NFKB1. In addition, the results showed Pi-cation, Pi-donor-hydrogen and Pi-alkyl were the main forms of interaction between scutellarin and NFKB1 and NFKBIA, Pi-Pi T-shaped, Pi-alkyl and hydrogen bonding were the main forms of interaction between scutellarin and RELA.

\section{Conclusion}

Taken together, TNF signaling pathway, NOD-like receptor signaling pathway and HIF-1 signaling pathway were possible signaling pathways, NFKBIA, RELA and NFKB1 were possible targets associated with the activities of scutellarin against osteoarthritis. However, it is imperative that these targets should be thoroughly verified by in vitro and in vivo experiments.

\section{Introduction}

Osteoarthritis (OA) is a chronic degenerative joint disease among the aged, and a major cause of severe joint pain and disability[1], which is characteristic of articular cartilage destruction, subchondral bone rebuilding and inflammation of the synovium $[2,3]$. Although the understanding of OA has been improved in recent years, there is still lack of effective drugs to delay and reverse the pathological process of $O A$, so that patients in advanced stages have to accept arthroplasty, which cause heavy burdens on society. Hence, it is urgent to find a new effective and low-cost medicine for OA[4, 5]. Previous studies have shown that the development of $\mathrm{OA}$ is closely related to aging, obesity, abnormal metabolism, osteoporosis, and joint malformation [6, 7]. Therefore, an effective treatment of OA must consider all these factors and the molecular networks behind them.

Scutellarin (SCU) (4',5,6-trihydroxy flavonoid-7-glucuronide), a flavonoid glycoside isolated from Erigeron breviscapus, exhibits anti-inflammatory, anti-oxidative, and anti-apoptotic activity in various pathological processes, including cerebrovascular disease, dermatitis, atherosclerosis, and myocardial infarction[8- 
10]. Recently, several studies have showed Scutellarin exerted inhibitive effects on the OA[11-13]. The underlying anti-OA mechanisms played by Scutellarin are closely related with several biological signal pathways, including Nrf2 pathway, Wnt/ $\beta$-catenin pathway, MAPK pathway, NF-KB pathway and PI3K/AKT pathway[11-13]. However, the detailed mechanisms of scutellarin against OA remain unclear.

With the development of bioinformatics, the study of disease processions and molecular mechanisms by constructing and detecting biological network is considered as a novel method that can effectively elucidate mechanism of action, which provides a new option for strategy transformation from one-target to multiple-target and for the discovery of new therapeutic targets[14, 15].

In present study, we investigated potential mechanisms of Scutellarin against OA by using network pharmacology and molecular docking. Our results may be helpful for systematic clarification of molecular mechanisms of Scutellarin against OA and facilitate the development of Scutellarin as a novel drug for $O A$.

\section{Materials And Methods}

\subsection{Predicting potential targets of scutellarin against OA}

Targets corresponding with scutellarin were collected from the following databases. GenCLiP3[16] (http://ci.smu.edu.cn/genclip3/analysis.php), PharmMapper[17] (http://www.lilabecust.cn/pharmmapper/), Swiss Target Prediction[18] (http://www.swisstargetprediction.ch/). The 'scutellarin' was submitted to search the related targets in GenCLiP3 database. The SDF format of Scutellarin (PubChem CID: 185617) was imputed to PharmMapper to obtain targets using the pharmacophore mapping approach. And in Swiss Target Prediction, we use SMILES string of scutellarin $(C 1=C C(=C C=C 1 C 2=C C(=0) C 3=C(C(=C(C=C 302) O C 4 C(C(C(C(04) C(=0) 0) 0) 0) 0) 0) 0) 0)$ to get the targets. There were 439 targets associated with scutellarin after removing repeat targets, and the specific information about genes was shown in supplementary Table 1. Genes related to OA were derived from the GenCLiP3 databases. 'Osteoarthritis' was used as the search term. There were 1594 targets in final list of genes linked with $\mathrm{OA}$ after deleting duplicates, and the information regarding these targets is provided in supplementary Table 2.

\subsection{Construction of PPI network and topological analysis}

Targets associated with scutellarin and OA collected from the various major databases were intersected to get the predicted common genes. The interaction network of common genes was constructed by using String database[19] and visualized in the Cytoscape3.7.2[20]. Network analyzer in Cytoscape was used to analyze topological parameters in PPI network. Then, the core targets were screened according to degree, with the upper limit of the screening range was the maximum degree value, and the lower limit was twice the median degree value.

\subsection{Cluster analysis}


Molecular Complex Detection Algorithm (MCODE), a software in Cytoscape3.7.2, was used to identify the important cluster in this PPI interaction network of 138 genes, which can also detect densely connected regions in PPI networks[21]. The criteria settings are degree cutoff $=2$, node score cutoff $=0.2, \mathrm{~K}$-core $=2$ and Max depth $=100$ to acquire the most valuable cluster. Afterward, the core modules networks were obtained.

\subsection{Genotype-phenotype correlation analysis}

VarElect[22] was used to explore the correlation between the predicted core targets and OA, which can quickly identify genes directly or indirectly related to a certain phenotype/disease and rank the genes.

\subsection{Biological functions analysis}

DAVID[23] was used to perform pathway enrichment analysis and R software (R3.6.3 for Windows, ggplot2 package) was used to visualize the core terms. Interaction networks between signal pathways was constructed by Cytoscape.

\subsection{Molecular docking}

In order to obtain the internal specific relationship, molecular docking was performed to assess the strength and mode of interactions between Scutellarin and the predicted targets. RCSB Protein Data Bank(PDB:2AZ5, 1SVC, 6Y1G,3QXY) were used to search the crystal structures of hub protein which may be the most potential targets of Scutellarin against Osteoarthritis, and then we remove waters, add hydrogen and patching to modify the crystal structure by Autodock[24, 25]. The Scutellarin structure was downloaded from PubChem, and the three-dimensional chemical structure was obtained by ChemBioDraw3D. Then, the molecular docking of Scutellarin and the hub targets were done by Autodock Vina[26] and Discovery Studio software, and visualized by PyMOL. Meanwhile, the affinity and the mode of action were analyzed.

\section{Results}

\subsection{Collection of candidate targets of scutellarin against OA}

The workflow diagram of this study was shown in Fig. 1. As results, 1594 OA associated genes were collected by using GenCLiP3 database. In addition, a total of 164 predicted targets were screened from GenCLiP3 database, 207 predicted targets were harvested from PharmMapper database and 101 predicted targets were gained from Swiss Target Prediction database. Finally, 171 candidate targets of scutellarin against OA were collected (Fig. 2).

\subsection{Construction of PPI network and topological analysis}

Further, 171 candidate proteins were used for the following construction of PPI networks. Under the confidence score above $0.9,138$ nodes and 761 edges were identified by String database, and the protein network was visualized by using Cytoscape (Fig. 3). 


\subsection{Topological network analysis and identification of core targets}

Network analyzer in Cytoscape software was used to assay the topological parameters of the PPI. The calculated median degree was 8 , and the maximum degree was 47 . Therefore, the degree in the screening conditions of core targets were ranged from 16 to 47 . As results, the 34 core target proteins were obtained and PPI network was visualized (Fig. 4).

\subsection{Correlation analysis between candidate targets and Osteoarthritis}

The 34 core genes were input into the VarElect to analyze genotype-phenotype correlations. As shown in Table 1, the 33 genes have the direct correlation with Osteoarthritis, IL1B, TNF, IL6, MMP9 and VEGFA have best correlation in these gene, with the score is $26.8,25.19,24.62,18.65$ and 10.75 , respectively. 
Table 1

VarElect analysis of targets of scutellarin with the OA phenotype

\begin{tabular}{|c|c|c|c|c|}
\hline NO. & Symbol & Description & Direct/Indirect & Score \\
\hline 1 & IL1B & Interleukin 1 Beta & Direct & 26.8 \\
\hline 2 & TNF & Tumor Necrosis Factor & Direct & 25.19 \\
\hline 3 & IL6 & Interleukin 6 & Direct & 24.62 \\
\hline 4 & MMP9 & Matrix Metallopeptidase 9 & Direct & 18.65 \\
\hline 5 & VEGFA & Vascular Endothelial Growth Factor A & Direct & 10.75 \\
\hline 6 & TP53 & Tumor Protein P53 & Direct & 10.7 \\
\hline 7 & ESR1 & Estrogen Receptor 1 & Direct & 9.17 \\
\hline 8 & JUN & $\begin{array}{l}\text { Jun Proto-Oncogene, AP-1 Transcription Factor } \\
\text { Subunit }\end{array}$ & Direct & 8.11 \\
\hline 9 & CCND1 & Cyclin D1 & Direct & 7.5 \\
\hline 10 & MAPK8 & Mitogen-Activated Protein Kinase 8 & Direct & 7.25 \\
\hline 11 & MAPK1 & Mitogen-Activated Protein Kinase 1 & Direct & 7.15 \\
\hline 12 & IL2 & Interleukin 2 & Direct & 7.13 \\
\hline 13 & CASP3 & Caspase 3 & Direct & 6.36 \\
\hline 14 & NFKB1 & Nuclear Factor Kappa B Subunit 1 & Direct & 6.35 \\
\hline 15 & FOS & $\begin{array}{l}\text { Fos Proto-Oncogene, AP-1 Transcription Factor } \\
\text { Subunit }\end{array}$ & Direct & 6.23 \\
\hline 16 & RELA & RELA Proto-Oncogene, NF-KB Subunit & Direct & 5.93 \\
\hline 17 & STAT3 & Signal Transducer And Activator Of Transcription 3 & Direct & 5.56 \\
\hline 18 & MAPK14 & Mitogen-Activated Protein Kinase 14 & Direct & 5.53 \\
\hline 19 & NFKBIA & NFKB Inhibitor Alpha & Direct & 5.33 \\
\hline 20 & CASP8 & Caspase 8 & Direct & 5.26 \\
\hline 21 & PIK3CA & $\begin{array}{l}\text { Phosphatidylinositol-4,5-Bisphosphate 3-Kinase } \\
\text { Catalytic Subunit Alpha }\end{array}$ & Indirect & 5.18 \\
\hline 22 & HRAS & HRas Proto-Oncogene, GTPase & Direct & 4.88 \\
\hline 23 & CDKN1A & Cyclin Dependent Kinase Inhibitor 1A & Direct & 4.8 \\
\hline 24 & MYC & MYC Proto-Oncogene, BHLH Transcription Factor & Direct & 4.55 \\
\hline
\end{tabular}




\begin{tabular}{|llllc|}
\hline NO. & Symbol & Description & Direct/lndirect & Score \\
\hline 25 & AKT1 & AKT Serine/Threonine Kinase 1 & Direct & 4.5 \\
\hline 26 & ITGB3 & Integrin Subunit Beta 3 & Direct & 4.27 \\
\hline 27 & BCL2 & BCL2 Apoptosis Regulator & Direct & 4.1 \\
\hline 28 & HSP90AA1 & $\begin{array}{l}\text { Heat Shock Protein 90 Alpha Family Class A Member } \\
1\end{array}$ & Direct & 3.82 \\
\hline 29 & EP300 & E1A Binding Protein P300 & Direct & 3.72 \\
\hline 30 & MAPK3 & Mitogen-Activated Protein Kinase 3 & Direct & 3.22 \\
\hline 31 & APP & Amyloid Beta Precursor Protein & Direct & 3.06 \\
\hline 32 & JAK2 & Janus Kinase 2 & Direct & 3.06 \\
\hline 33 & EGFR & Epidermal Growth Factor Receptor & Direct & 2.62 \\
\hline 34 & CREB1 & CAMP Responsive Element Binding Protein 1 & Direct & 2.39 \\
\hline $\begin{array}{l}\text { The 34 genes were divided into two parts that are directly related and indirectly related to "OA". The } \\
\text { score indicates the correlation between genes and the target phenotype }\end{array}$ & \\
\hline 3
\end{tabular}

\subsection{Clustering analysis in PPI network}

Next, three dominant clustering subnetworks were obtained from 138 candidate targets by using MCODE algorithm in Cytoscape software (Fig. 5A). 15 core targets were included in cluster 1, mainly associated with TNF signaling pathway, PI3K-Akt signaling pathway, HIF-1 signaling pathway, Toll-like receptor signaling pathway. 10 core targets were involved in cluster 2, mainly involved in PI3K-Akt signaling pathway, NOD-like receptor signaling pathway, TNF signaling pathway, HIF-1 signaling pathway and JAKSTAT signaling. 6 core targets were present in cluster 3, mainly related with MAPK signaling pathway, NOD-like receptor signaling pathway and PI3K-Akt signaling pathway (Fig. 5B).

\subsection{KEGG enrichment analysis of core targets of scutellarin against $O A$}

Enrichment analysis was performed by DAVID, as shown in Fig. 6. KEGG enrichment analysis indicated that 34 core targets of scutellarin against OA were mainly enriched in several kinds of pathways including TNF signaling pathway, PI3K-Akt signaling pathway, NOD-like receptor signaling pathway, Toll-like receptor signaling pathway, and HIF-1 signaling pathway (Fig. 6).

\subsection{Molecular docking of scutellarin and its potential targets}


To further explain the inner relationship between scutellarin and its predicted targets related to $O A$, the key genes of NF-kB signaling pathway including NFKBIA, NFKB1 and RELA, which were the key link of Hypoxia-inducible factor-1, NOD-like receptors and TNF-a signaling pathway, were selected to perform molecular docking. The results showed that there was better interaction between scutellarin and its potential targets, with the binding energy was-7.3 kcal/mol (NFKB1), $-8.3 \mathrm{kcal} / \mathrm{mol}$ (NFKBIA), $-7.2 \mathrm{kcal} / \mathrm{mol}$ (RELA), respectively (Fig. 7). In addition, the results showed Pi-cation, Pi-donor-hydrogen and Pi-alkyl were the main forms of interaction between scutellarin and NFKB1(1SVC) and NFKBIA(6Y1J), Pi-Pi T-shaped, Pi-alkyl and hydrogen bonding were the main forms of interaction between scutellarin and RELA(3QXY).

\section{Discussion}

OA, a biomechanical disease, which is estimated to be the fourth leading cause of disability by $2020[27$, 28]. Although there are already several drugs for $O A$ in clinical practice, including sodium hyaluronate, glucosamine, chondroitin sulphate, and NASIDs, most of them were not proved to truly prevent the progression of $O A$, and many side effects were appeared in application[29, 30], which may be due to the complicated pathological mechanism of $\mathrm{OA}$, and the single-target drugs currently used in clinical practice could not fully prevent the progression of OA. Thus, our present study is trying to clarify the molecular mechanism of Scutellarin against OA by network pharmacology, aim to facilitate the development of Scutellarin, a safer agent derived natural plant, as a novel drug for $\mathrm{OA}$, and promote development of $\mathrm{OA}$ therapy with natural plant derived agent mediated multi-target therapeutics. We collected information from public databases to predict potential targets of Scutellarin against OA, as well as to analyze the key signaling pathways and hub genes involved in the role of Scutellarin against OA. We also performed molecular docking to predict specific binding sites between Scutellarin and its predicted targets.

Firstly, we obtained the potential targets of Scutellarin against OA by using public database, and finally got 171 targets, which may be the important targets involved in the action of Scutellarin against OA. Then, we screened 34 core targets from 171 targets by degree score. Meanwhile, we performed cluster analysis and got three core clusters, which were principally linked to TNF signaling pathway, PI3K-Akt signaling pathway, NOD-like receptor signaling pathway, Toll-like receptor signaling pathway, HIF-1 signaling pathway, JAK-STAT signaling pathway and MAPK signaling pathway. In addition, we also found 34 core target genes were mainly enriched in TNF signaling pathway, PI3K-Akt signaling pathway, NOD-like receptor signaling pathway, Toll-like receptor signaling pathway, and HIF-1 signaling pathway. These data indicated scutellarin might play the inhibitive effect on OA by regulating these signaling pathway. Previous reports showed scutellarin could regulate PI3K-Akt signaling pathway, MAPK signaling pathway, Wnt/ $\beta$-catenin signaling pathway and TLR4 signaling pathway, thus exerted the inhibitive action on OA progression. So, our data were partly in accordance with previous data. In addition, we also identified new signaling pathways including TNF signaling pathway, NOD-like receptor signaling pathway and HIF-1 signaling pathway, which were all the core signaling pathway in the pathogenesis of OA[3136], thus may be involved in the inhibitive effect of Scutellarin on OA. Our data will be the supplement for the present studies on the molecular mechanisms of Scutellarin against OA. As we know, NF-kB signaling 
pathway was the key link of Hypoxia-inducible factor-1, NOD-like receptors and TNF-a induced OA progression [34, 36, 37]. NF-kB signaling pathway was also the key target of the several anti-osteoarthritis compounds, including Scutellarin $[11,38,39]$. However, the action mode of Scutellarin on NF-kB signaling pathway was still unknown. In our study, we found several key factors including NFKBIA, RELA and NFKB1 were involved in the 34 core targets of Scutellarin against OA. So, we next performed the molecular docking to clarify the action mode of scutellarin on NF-kB signaling pathway. We found there are better docking between Scutellarin and three key factors of NF-kB signaling pathway, indicating scutellarin may bind directly with NFKBIA, RELA and NFKB1, thus inhibit the activity of NF-kB signaling pathway, finally play inhibitive effect on OA. Based on the literature and our data, several signaling pathways including PI3K-Akt signaling pathway, MAPK signaling pathway, Wnt/ $\beta$-catenin signaling pathway and TLR4 signaling pathway, TNF signaling pathway, NOD-like receptor signaling pathway and HIF-1 signaling pathway were possible signaling pathways involved in the activities of Scutellarin against OA. Among these, TNF signaling pathway, NOD-like receptor signaling pathway and HIF-1 signaling pathway were newly identified signaling pathway for clarification of the mechanisms of Scutellarin against OA. In addition, NFKBIA, RELA and NFKB1, the key factors of NF-kB signaling pathway, may be the key targets associated with the activities of Scutellarin against OA by TNF signaling pathway, NODlike receptor signaling pathway and HIF-1 signaling pathway.

Our study had several limitations. Firstly, the data set of the OA-related genes we used was based on existing research findings, which hinders the discovery of new targets for OA. Secondly, based on the topological analysis and key targets analysis of the constructed network, we only predicted the possible targets and signaling pathways associated with the activities of Scutellarin against OA. In the following study, we should verify the effect of Scutellarin on these key targets and signaling pathways by further biological experiments.

Overall, the current study found TNF signaling pathway, NOD-like receptor signaling pathway and HIF-1 signaling pathway were new possible signaling pathways and NFKBIA, RELA and NFKB1 were new possible targets associated with the activities of scutellarin against $O A$ by a systematic network pharmacology and molecular docking. However, it is imperative that these targets should be thoroughly verified by in vitro and in vivo experiments.

\section{Declarations}

\section{Authors' contributions}

Mingli Sun and Xinan Zhang dominate the concept and carry out a comprehensive design, Shijia Guo is responsible for network pharmacology analysis and molecular docking. all authors participated in the analysis and interpretation of data and approved the final paper.

\section{Funding}


This work is supported by the Innovative Talents Support Program for Universities of Liaoning Province (WR2019024) and Scientific Research Project on Young Scientific and Technological Talents of Department of Education of Liaoning Province (QN2019033).

\section{Availability of data and materials}

The additional data can be found in supplementary materials.

\section{Ethics approval and consent to participate}

Not applicable

\section{Consent for publication}

Not applicable

\section{Competing interests}

There is no conflict of interest to declare.

\section{References}

1. Lane NE, Shidara K, Wise BL. Osteoarthritis year in review 2016: clinical. Osteoarthritis Cartilage. 2017;25(2):209-15.

2. Loeser RF. Aging and osteoarthritis: the role of chondrocyte senescence and aging changes in the cartilage matrix. Osteoarthritis Cartilage. 2009;17(8):971-9.

3. Glyn-Jones S, Palmer AJR, Agricola R, Price AJ, Vincent TL, Weinans H, et al. Osteoarthritis The Lancet. 2015;386(9991):376-87.

4. Bellamy N, Campbell J, Robinson V, Gee T, Bourne R, Wells G. Intraarticular corticosteroid for treatment of osteoarthritis of the knee. Cochrane Database Syst Rev. 2006(2):CD005328.

5. Troeberg L, Nagase H. Proteases involved in cartilage matrix degradation in osteoarthritis. Biochim Biophys Acta. 2012;1824(1):133-45.

6. Goldring MB, Goldring SR. Osteoarthritis. J Cell Physiol. 2007;213(3):626-34.

7. Blagojevic M, Jinks C, Jeffery A, Jordan KP. Risk factors for onset of osteoarthritis of the knee in older adults: a systematic review and meta-analysis. Osteoarthritis Cartilage. 2010;18(1):24-33.

8. Mo J, Yang R, Li F, Zhang X, He B, Zhang Y, et al. Scutellarin protects against vascular endothelial dysfunction and prevents atherosclerosis via antioxidation. Phytomedicine. 2018;42:66-74.

9. Sun JB, Li Y, Cai YF, Huang Y, Liu S, Yeung PK, et al. Scutellarin protects oxygen/glucose-deprived astrocytes and reduces focal cerebral ischemic injury. Neural Regen Res. 2018;13(8):1396-407.

10. Wang L, Ma Q. Clinical benefits and pharmacology of scutellarin: A comprehensive review. Pharmacol Ther. 2018;190:105-27. 
11. Wang W, Li J, Li F, Peng J, Xu M, Shangguan Y, et al. Scutellarin suppresses cartilage destruction in osteoarthritis mouse model by inhibiting the NF-KB and PI3K/AKT signaling pathways. Int Immunopharmacol. 2019;77:105928.

12. Liu F, Li L, Lu W, Ding Z, Huang W, Li YT, et al. Scutellarin ameliorates cartilage degeneration in osteoarthritis by inhibiting the Wnt/beta-catenin and MAPK signaling pathways. Int Immunopharmacol. 2020;78:105954.

13. Luo Z, Hu Z, Bian Y, Su W, Li X, Li S, et al. Scutellarin Attenuates the IL-1beta-Induced Inflammation in Mouse Chondrocytes and Prevents Osteoarthritic Progression. Front Pharmacol. 2020;11:107.

14. Yang M, Chen JL, Xu LW, Ji G. Navigating traditional chinese medicine network pharmacology and computational tools. Evid Based Complement Alternat Med. 2013;2013:731969.

15. Luo TT, Lu Y, Yan SK, Xiao X, Rong XL, Guo J. Network Pharmacology in Research of Chinese Medicine Formula: Methodology, Application and Prospective. Chin J Integr Med. 2020;26(1):72-80.

16. Wang JH, Zhao LF, Wang HF, Wen YT, Jiang KK, Mao XM, et al. GenCLiP 3: mining human genes' functions and regulatory networks from PubMed based on co-occurrences and natural language processing. Bioinformatics. 2019.

17. Wang X, Shen Y, Wang S, Li S, Zhang W, Liu X, et al. PharmMapper 2017 update: a web server for potential drug target identification with a comprehensive target pharmacophore database. Nucleic Acids Res. 2017;45(W1):W356-W60.

18. Daina A, Michielin O, Zoete V. SwissTargetPrediction: updated data and new features for efficient prediction of protein targets of small molecules. Nucleic Acids Res. 2019;47(W1):W357-W64.

19. Szklarczyk D, Gable AL, Lyon D, Junge A, Wyder S, Huerta-Cepas J, et al. STRING v11: protein-protein association networks with increased coverage, supporting functional discovery in genome-wide experimental datasets. Nucleic Acids Res. 2019;47(D1):D607-D13.

20. Otasek D, Morris JH, Boucas J, Pico AR, Demchak B. Cytoscape Automation: empowering workflowbased network analysis. Genome Biol. 2019;20(1):185.

21. Bader GD, Hogue CW. An automated method for finding molecular complexes in large protein interaction networks. BMC Bioinformatics. 2003;4:2.

22. Stelzer G, Plaschkes I, Oz-Levi D, Alkelai A, Olender T, Zimmerman S, et al. VarElect: the phenotypebased variation prioritizer of the GeneCards Suite. BMC Genomics. 2016;17 Suppl 2:444.

23. Huang da W, Sherman BT, Lempicki RA. Systematic and integrative analysis of large gene lists using DAVID bioinformatics resources. Nature protocols. 2009;4(1):44-57.

24. Morris GMGD, Halliday DS, Huey R, Hart WE, Belew R, Olson AJ. Automated Docking Using a Lamarckian Genetic Algorithm and an Empirical Binding Free Energy Function. J Comp Chem. 1998;19:1639-62.

25. Huey R, Morris GM, Olson AJ, Goodsell DS. A semiempirical free energy force field with charge-based desolvation. J Comput Chem. 2007;28(6):1145-52. 
26. Trott O, Olson AJ. AutoDock Vina: improving the speed and accuracy of docking with a new scoring function, efficient optimization, and multithreading. J Comput Chem. 2010;31(2):455-61.

27. Teirlinck CH, Dorleijn DMJ, Bos PK, Rijkels-Otters JBM, Bierma-Zeinstra SMA, Luijsterburg PAJ. Prognostic factors for progression of osteoarthritis of the hip: a systematic review. Arthritis Res Ther. 2019;21(1):192.

28. Thielen NGM, van der Kraan PM, van Caam APM. TGFbeta/BMP Signaling Pathway in Cartilage Homeostasis. Cells. 2019;8(9).

29. Bannuru RR, Osani MC, Vaysbrot EE, Arden NK, Bennell K, Bierma-Zeinstra SMA, et al. OARSI guidelines for the non-surgical management of knee, hip, and polyarticular osteoarthritis. Osteoarthritis Cartilage. 2019;27(11):1578-89.

30. Restaino OF, Finamore R, Stellavato A, Diana P, Bedini E, Trifuoggi M, et al. European chondroitin sulfate and glucosamine food supplements: A systematic quality and quantity assessment compared to pharmaceuticals. Carbohydrate Polymers. 2019;222.

31. Kim HW, Kwon YJ, Park BW, Song JJ, Park YB, Park MC. Differential expressions of NOD-like receptors and their associations with inflammatory responses in rheumatoid arthritis. Clin Exp Rheumatol. 2017;35(4):630-7.

32. Li H, Xie S, Qi Y, Li H, Zhang R, Lian Y. TNF-a increases the expression of inflammatory factors in synovial fibroblasts by inhibiting the PI3K/AKT pathway in a rat model of monosodium iodoacetateinduced osteoarthritis. Exp Ther Med. 2018;16(6):4737-44.

33. Fernández-Torres J, Martínez-Nava GA, Zamudio-Cuevas Y, Martínez-Flores K, Gutiérrez-Ruíz MC, Gómez-Quiroz LE, et al. Impact of the gene-gene interactions related to the HIF-1a signaling pathway with the knee osteoarthritis development. Clin Rheumatol. 2019;38(10):2897-907.

34. Hwang HS, Lee MH, Choi MH, Kim HA. NOD2 signaling pathway is involved in fibronectin fragmentinduced pro-catabolic factor expressions in human articular chondrocytes. BMB Rep. 2019;52(6):373-8.

35. Zhao Y, Li Y, Qu R, Chen X, Wang W, Qiu C, et al. Cortistatin binds to TNF-a receptors and protects against osteoarthritis. EBioMedicine. 2019;41:556-70.

36. Okada K, Mori D, Makii Y, Nakamoto H, Murahashi Y, Yano F, et al. Hypoxia-inducible factor-1 alpha maintains mouse articular cartilage through suppression of NF-KB signaling. Sci Rep. 2020;10(1):5425.

37. Yan L, Zhou L, Xie D, Du W, Chen F, Yuan Q, et al. Chondroprotective effects of platelet lysate towards monoiodoacetate-induced arthritis by suppression of TNF-a-induced activation of NF-KB pathway in chondrocytes. Aging. 2019;11(9):2797-811.

38. Chern CM, Zhou H, Wang YH, Chang CL, Chiou WF, Chang WT, et al. Osthole ameliorates cartilage degradation by downregulation of NF-KB and HIF-2a pathways in an osteoarthritis murine model. Eur J Pharmacol. 2020;867:172799.

39. Ma P, Yue L, Yang H, Fan Y, Bai J, Li S, et al. Chondroprotective and anti-inflammatory effects of amurensin H by regulating TLR4/Syk/NF-kappaB signals. J Cell Mol Med. 2020;24(2):1958-68. 


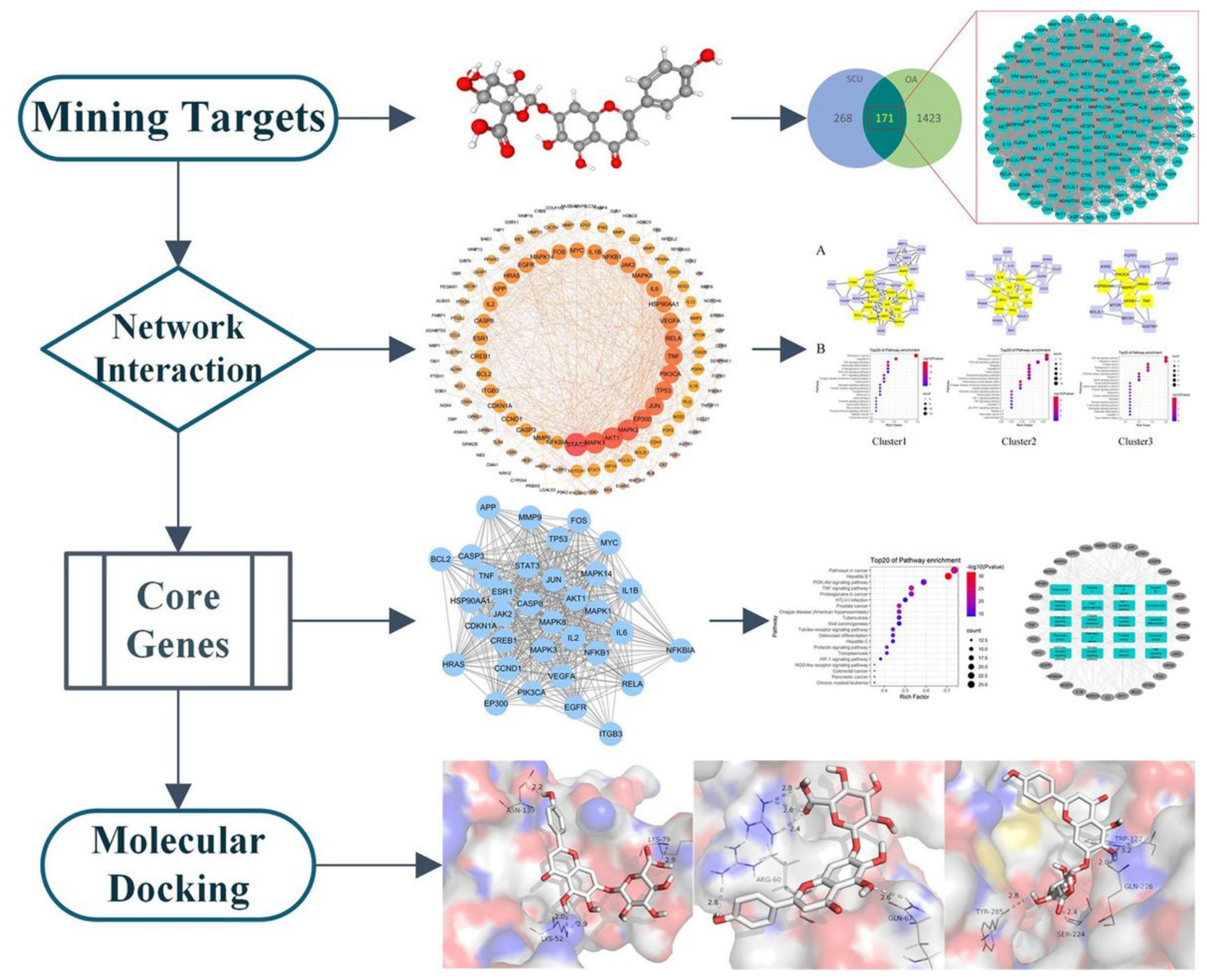

Figure 1

The workflow diagram for the prediction of potential targets of scutellarin against OA 


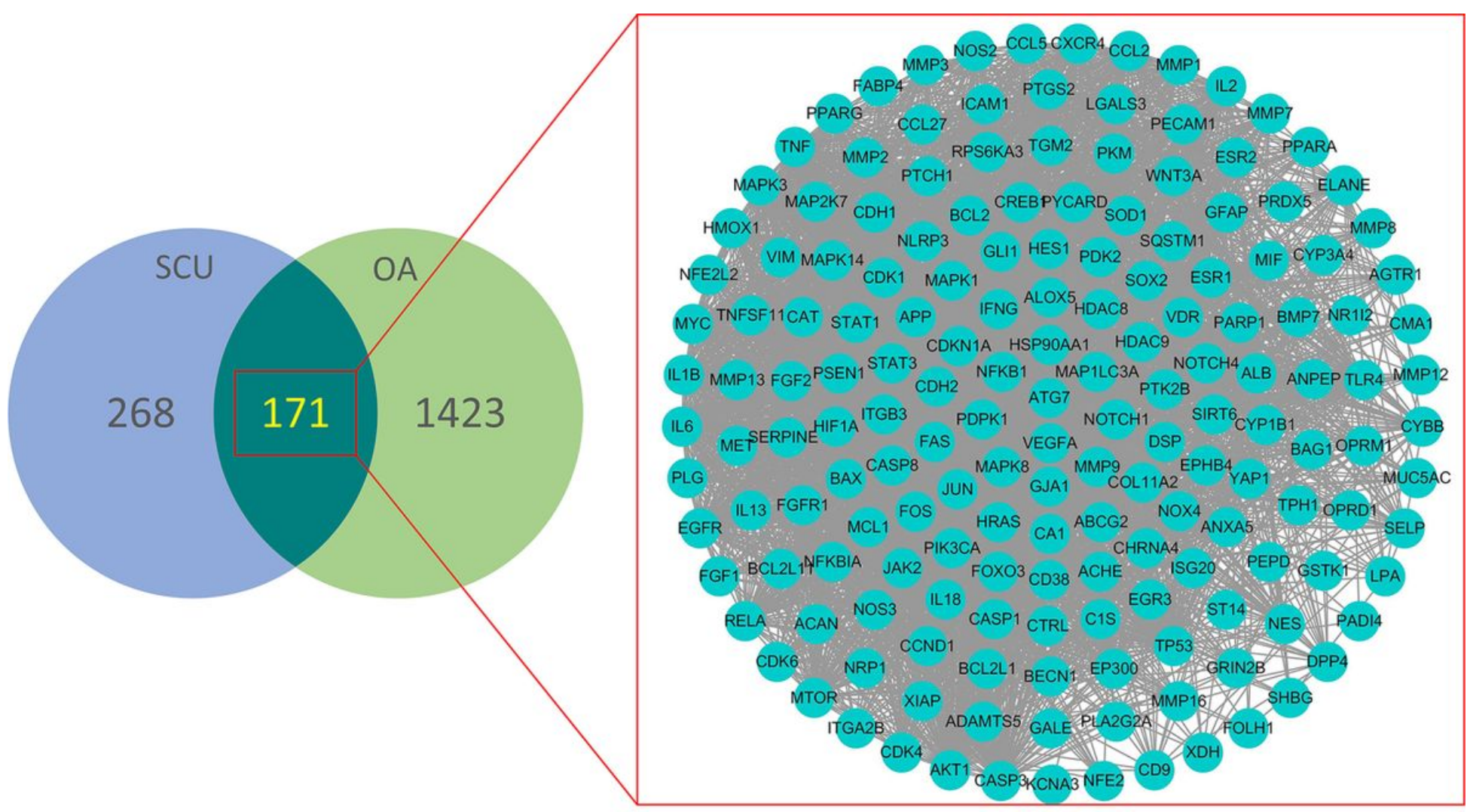

Figure 2

A venn diagram of predicted intersection genes between scutellarin and osteoarthritis 


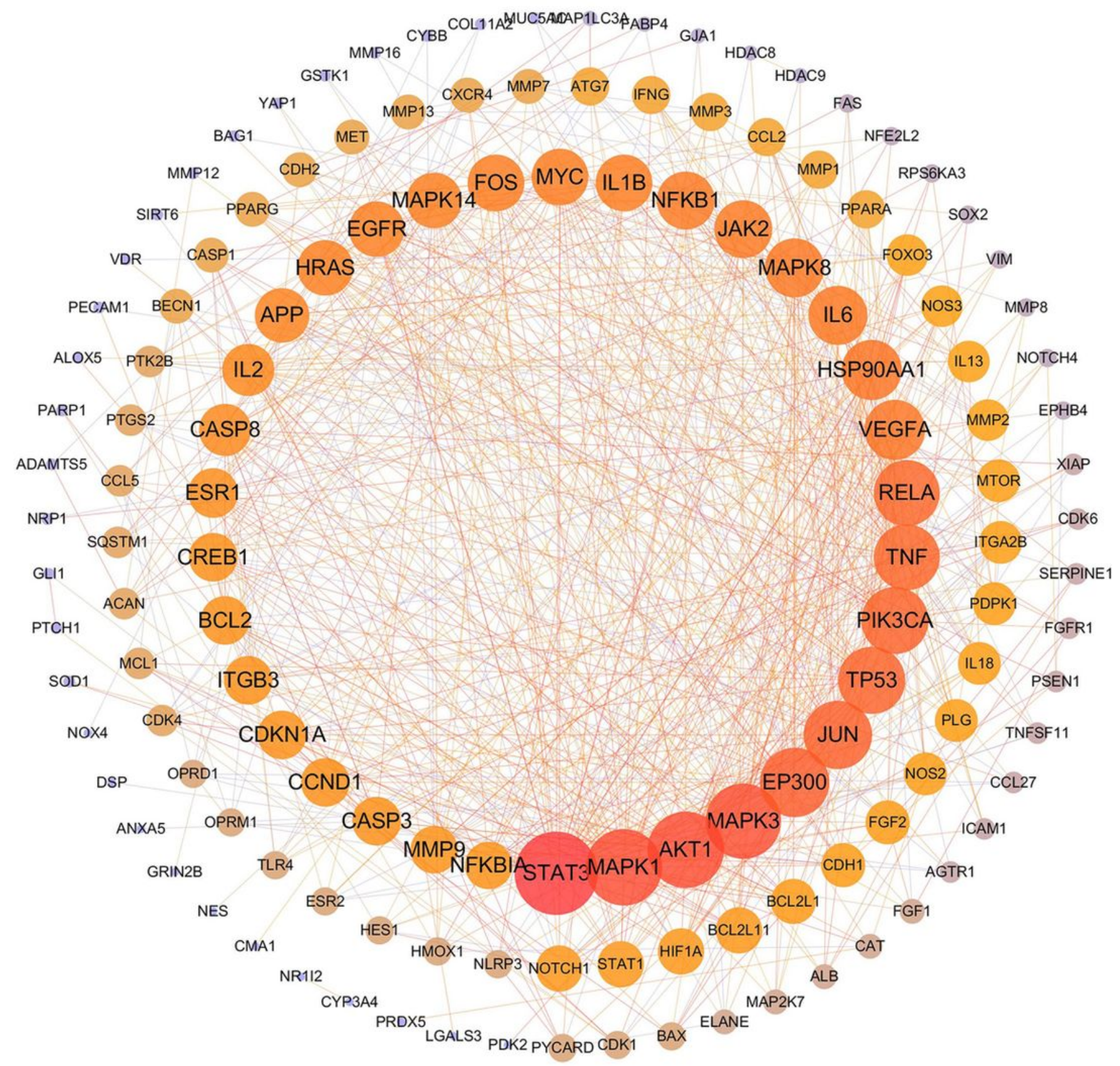

Figure 3

PPI network of potential targets of scutellarin against OA constructed by network analyzer tool. The larger the circle, the deeper the red color, the greater the degree. The closer the connection between the two nodes, the darker the color of edges and the thicker the line. 


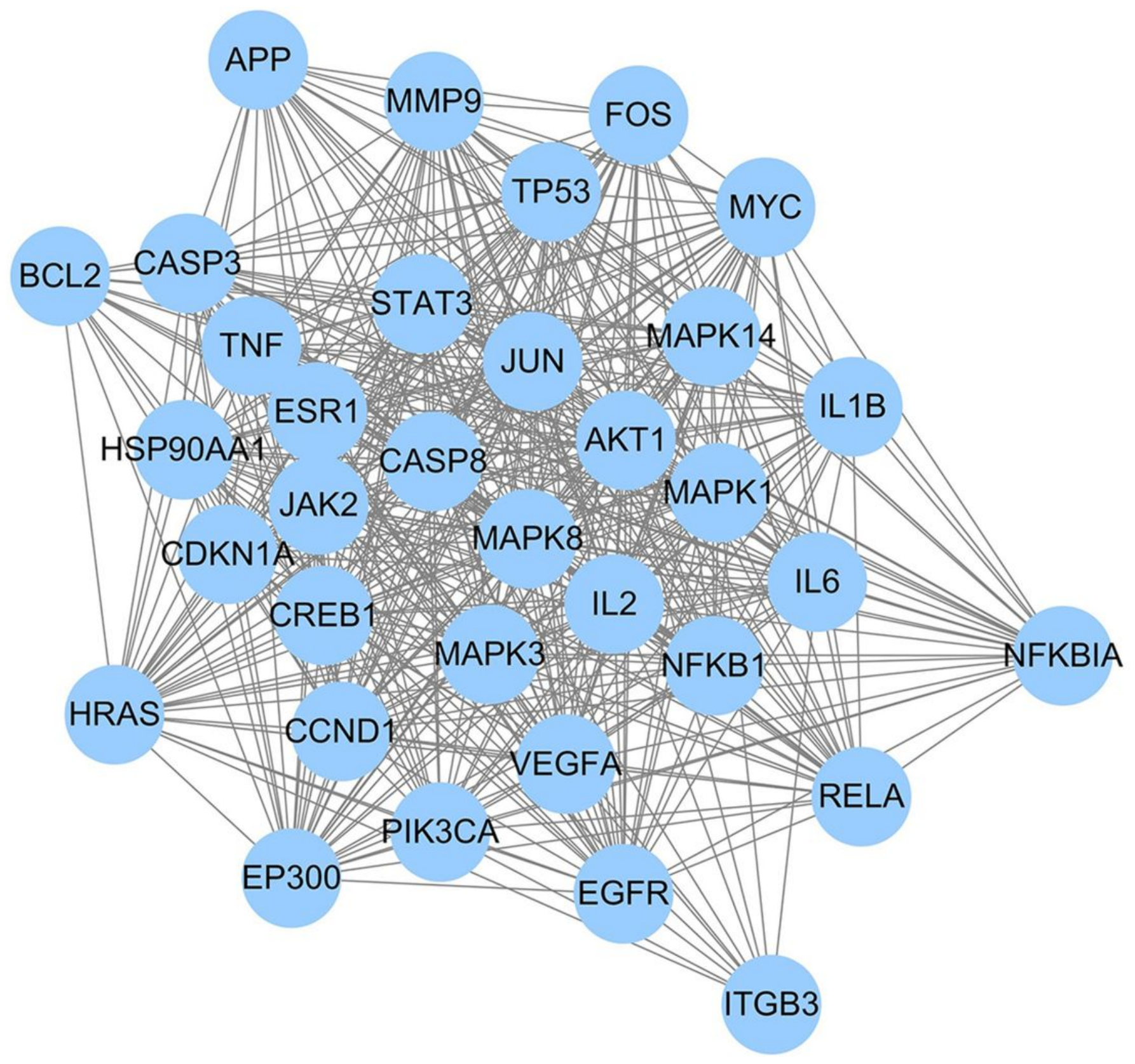

Figure 4

The 34 core targets screened according to degree value were visualized by Cytoscape. 

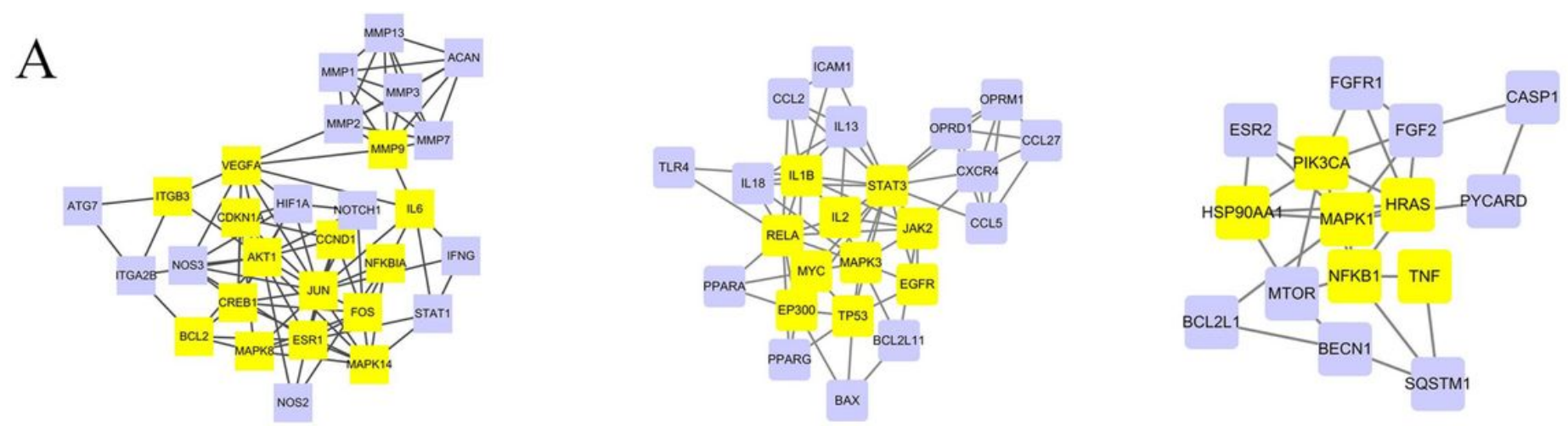

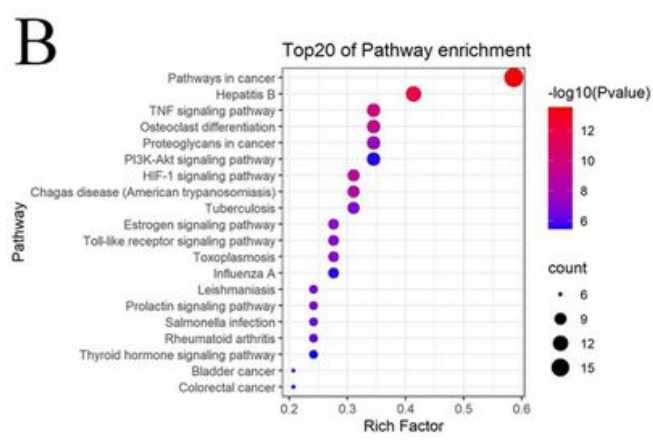

Cluster1

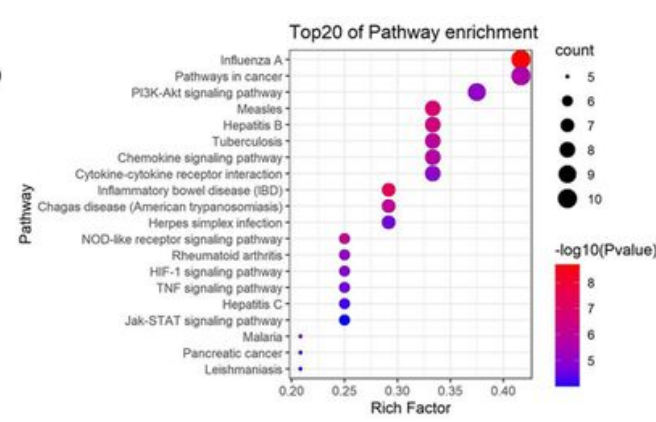

Cluster2

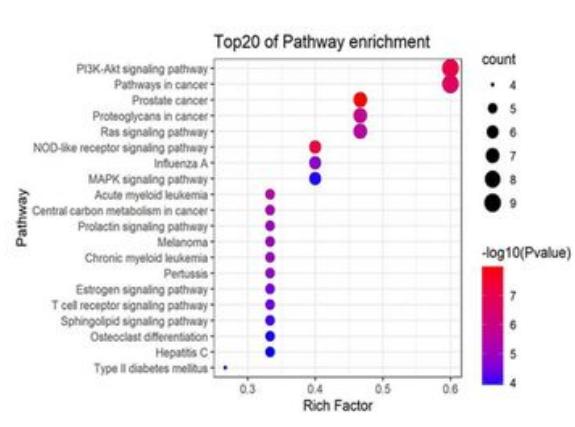

Cluster3

\section{Figure 5}

Cluster analysis and KEGG pathway enrichment of potential targets of scutellarin against OA. (A) Cluster analysis was done by MCODE algorithm based on the PPI networks of predicted targets, and the several core targets were clustered in different clusters. (B) The top 20 pathway enrichments of three clusters were analyzed by DAVID.

A

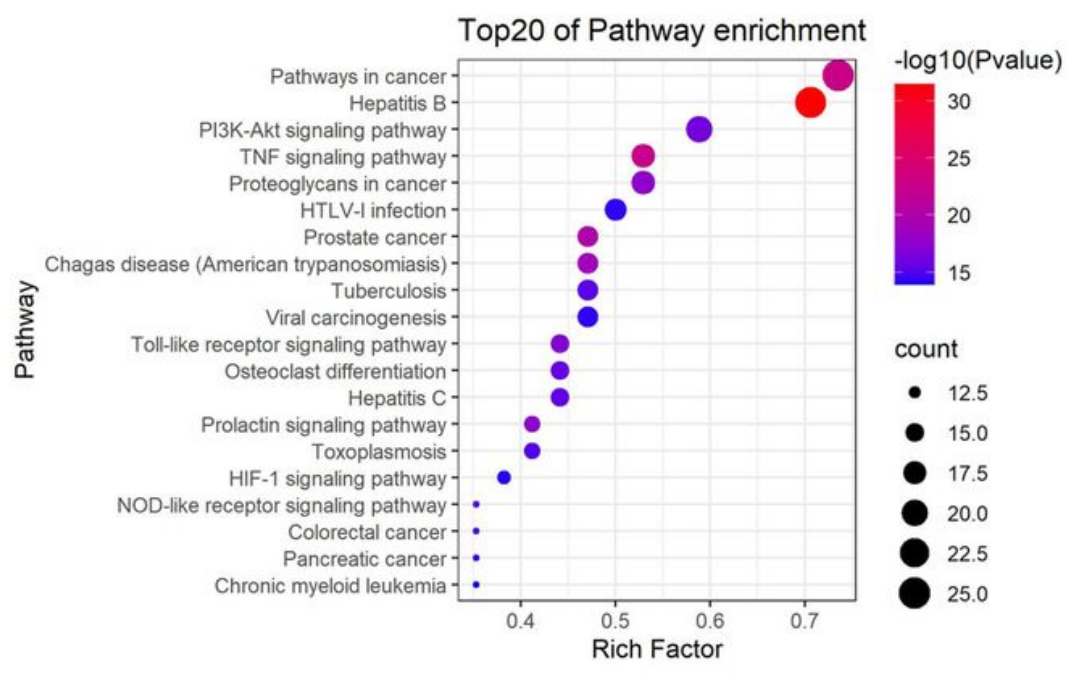

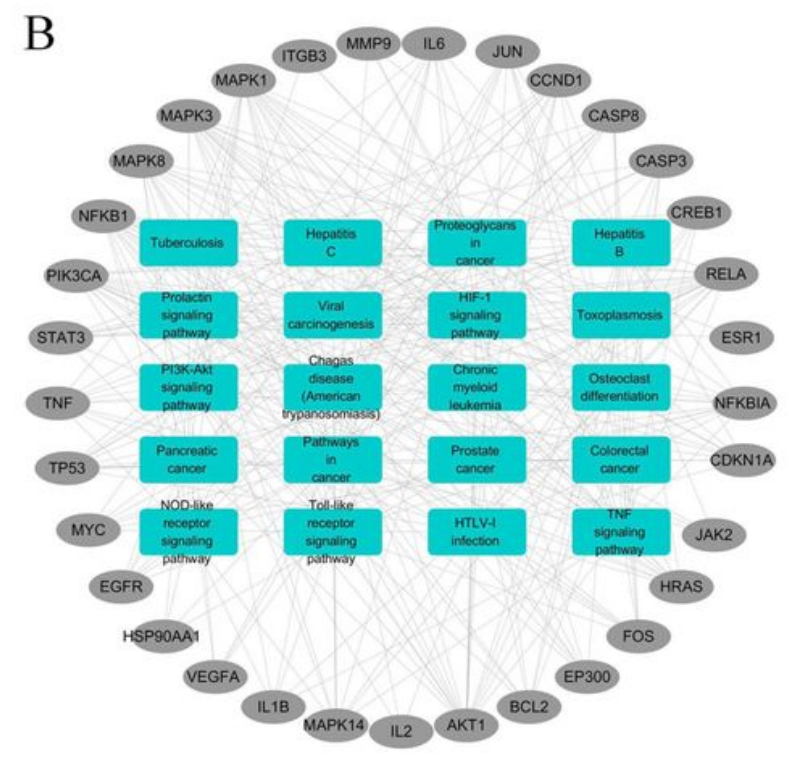

Figure 6 
Pathway enrichment of 34 core genes. The top 20 pathway enrichments of 34 core genes were analyzed by DAVID and visualized by R software (A), and Cytoscape(B).
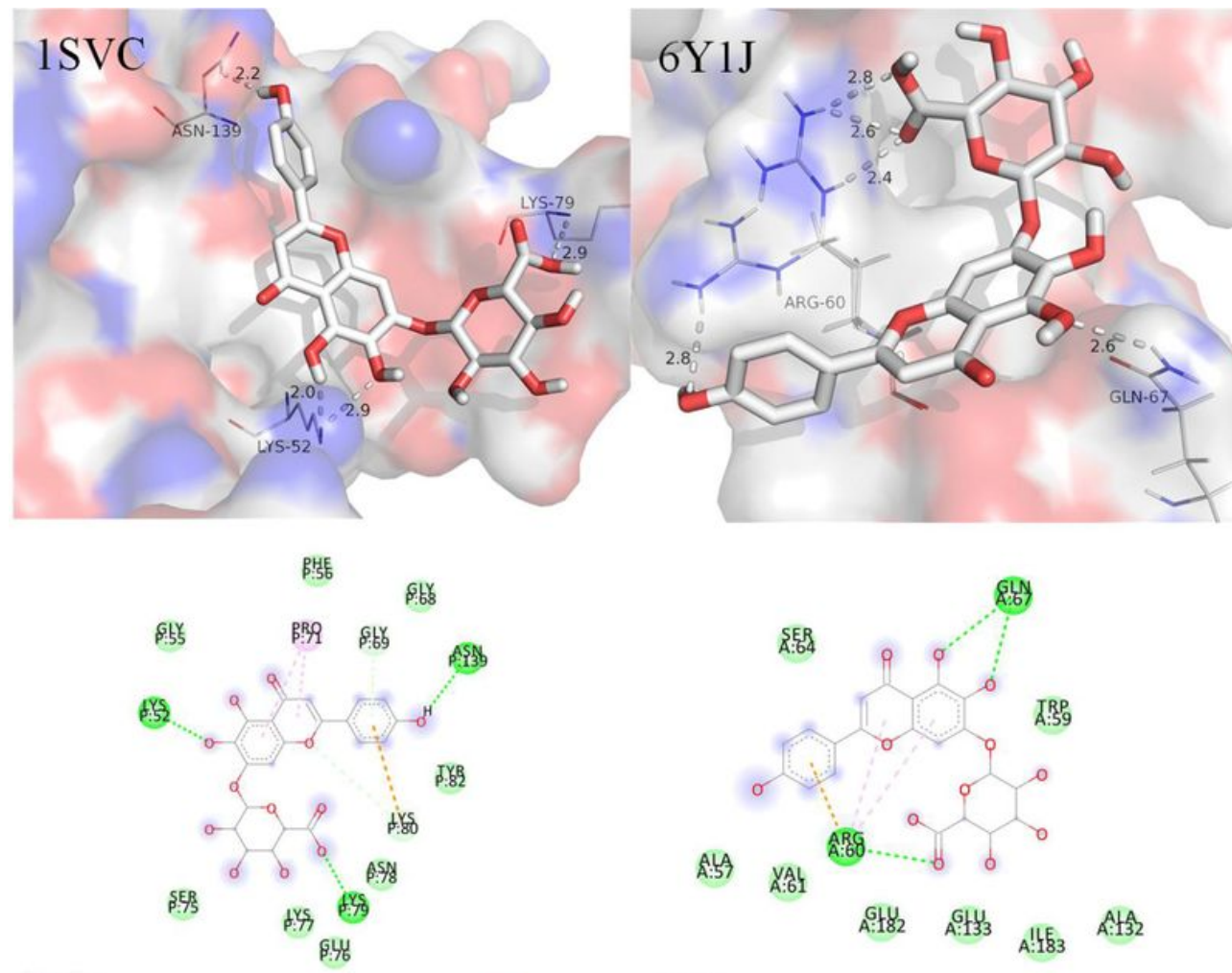

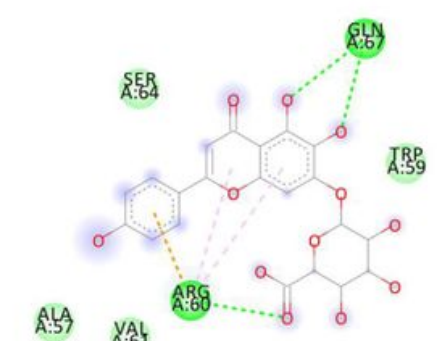

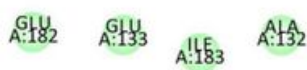
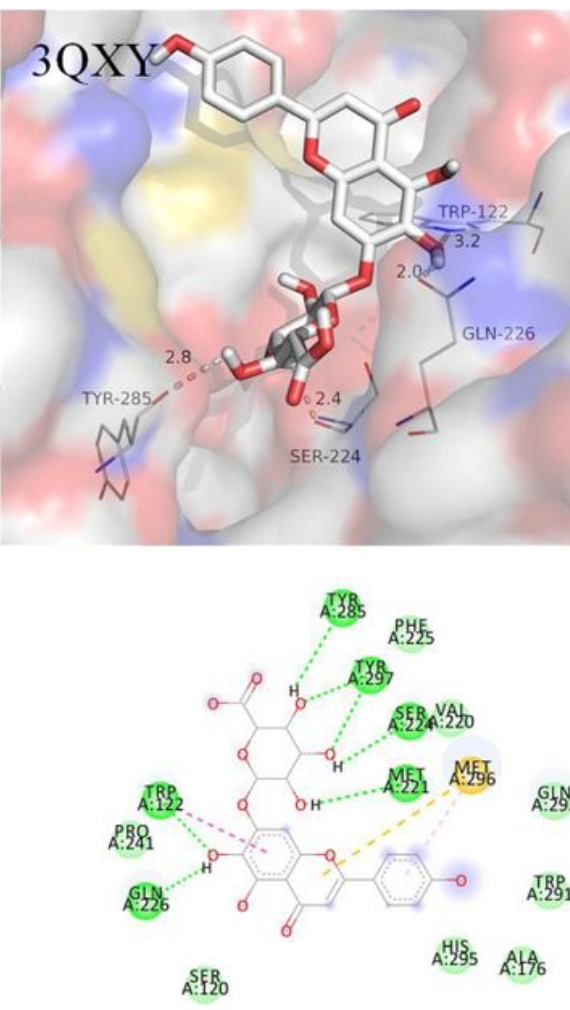

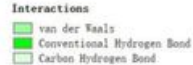
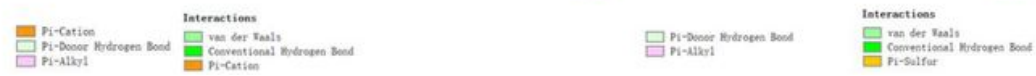

\section{Figure 7}

Molecular docking of scutellarin and its potential targets. The 3D and 2D presentation of molecular docking model between scutellarin and NFKB1(1SVC), NFKBIA(6Y1J) and RELA(3QXY). Scutellarin molecule was showed as a stick and residues were showed as lines in the binding sites. The hydrogen bonds, Pi-Pi T-shaped, Pi-Alkyl and Pi-cation were demarcated with dashed lines of different color, and the interaction distances were indicated next to the bonds.

\section{Supplementary Files}

This is a list of supplementary files associated with this preprint. Click to download.

- SupplementaryMaterial.docx 